\section{Proposta de residência em Atenção Domiciliar como área de atuação}

\author{
Proposal of Home Care Residency as actuation field \\ Propuesta de residencia en Atención Domiciliar como área de \\ actuación
}

\section{Resumo}

A Atenção Domiciliar (AD) configura-se numa área de atuação de diversos especialistas, como Médicos de Família e Comunidade, Geriatras, Pediatras e Internistas. Por suas especificidades e dentro do seu campo de práticas, demanda competências que não estão contempladas isoladamente em nenhuma especialidade médica, sendo que o tempo e a estrutura necessários para a aquisição destas ultrapassa a capacidade de inserção ao longo dos programas atuais. A expansão desta modalidade de cuidados representa uma demanda crescente de formação, exigindo a inclusão da AD como potencial campo de trabalho destes especialistas, concebendo a necessidade de um ano adicional para formação nas especificidades dessa área de atuação. Discutem-se as estratégias de formação, campos da prática e estrutura programática necessários para a consolidação desta proposta. Espera-se que este artigo possa subsidiar a natural construção desta especialidade no âmbito da atuação médica em saúde.

Palavras-chave: Internato e Residência; Serviços de Assistência Domiciliar; Visita Domiciliar; Educação em Saúde; Educação Médica

\begin{abstract}
Home Care $(\mathrm{HC})$ is an expertise area for several specialists, such as Family Physicians, Geriatricians, Pediatricians and Internal Medicine. Due to its specificities and within its practice field, it demands competences that are not contemplated apart in any medical specialty, and the time and structure required to acquire them surpasses the insertion capacity in the current programs. This care type expansion represents a growing demand for training, requiring $\mathrm{HC}$ inclusion as a potential field of work for these specialists, conceiving the need for an additional year for the formation of specific features as an activity area. We discuss training strategies, practice fields and programmatic structure required to consolidate this proposal. We expect that this article can subsidize the natural construction of this specialty within the scope of health medical practice.
\end{abstract}

Keywords: Internship and Residence; Home Care Services; Home Visit; Health Education; Education, Medical
Leonardo Cançado Monteiro Savassi ${ }^{a}$

Cibele Gomes Lima Melob

Daniel Almeida Gonçalves ${ }^{c}$

Denize Ornelas Pereira Salvador Oliveira ${ }^{d}$

Heitor Rossi Lopes ${ }^{e}$

Renata Luciana Hasegawa Fregonezi

Sara Turcotteg

a Universidade Federal de Ouro Preto (UFOP) Universidade Aberta do SUS (UNASUS). Ouro Preto, MG, Brasil. leosavassi@gmail.com (Autor correspondente)

b Programa de Residência em Medicina de Família e Comunidade da Casa de Saúde Santa Marcelina; MEMAD - Casa de Saúde Santa Marcelina e Associação Paulista para o Desenvolvimento da Medicina. São Paulo, SP, Brasil. cibellegomesmelo@yahoo.com.br

c Programa de Residência em Medicina de Família e Comunidade da Universidade Federal de São Paulo (UNIFESP)/Escola Paulista de Medicina. São Paulo, SP, Brasil. daniel.almeida.33@gmail.com

d Programa de Residência em Medicina de Família e Comunidade da Secretaria de Saúde de São Bernardo do Campo. São Bernardo do Campo, SP, Brasil. denizeofp@yahoo.com.br

e Faculdade de Medicina do Santa Marcelina (FASM); Programa de Residência em Medicina de Família e Comunidade do Hospital Santa Marcelina, da Universidade de Toronto (Canadá) - "Global Health and Vulnerable Populations" e do Internato Médico da FASM - Casa de Saúde Santa Marcelina; EMAD UBS Jardim São Carlos - Casa de Saúde Santa Marcelina. São Paulo, SP, Brasil. heitor_72@yahoo.com.br

' Programa de Residência em Medicina de Família e Comunidade da Secretaria Municipal de Saúde de São Paulo - $8^{\text {a }}$ Coreme - Residência em Rede. São Paulo, SP, Brasil. renatafregonezi@gmail.com

${ }^{9}$ Departamento de Medicina de Família da Associação Paulista de Medicina; Associação Paulista de Medicina de Família e Comunidade. São Paulo, SP, Brasil. sara@fraternidadeclinica.com.br

Fonte de financiamento: declaram não haver. Parecer CEP: não se aplica. Conflito de interesses: declaram não haver. Procedência e revisão por pares: revisado por pares. Recebido em: 27/08/2017. Aprovado em: 29/12/2017.
Como citar: Savassi LCM, Melo CGL, Gonçalves DA, Oliveira DOPS, Lopes HR, Fregonezi RLH, et al. Proposta de residência em Atenção Domiciliar como área de atuação. Rev Bras Med Fam Comunidade. 2018;13(40):1-13. http://dx.doi.org/10.5712/rbmfc13(40)1599 


\section{Resumen}

La Atención Domiciliaria (AD) se configura en un área de actuación de diversos especialistas, como Médicos de Familia, Geriatras, Pediatras y Medicina Interna. Debido a sus especificidades y dentro de su campo de práctica, exige competencias que no están contempladas aisladamente en cualquier especialidad médica, y el tiempo y la estructura necesaria para adquirirlas sobrepasa la capacidad de inserción en los programas actuales. La expansión de este tipo de atención representa una demanda creciente de capacitación, requiriendo la inclusión de la AD como un potencial campo de trabajo para estos especialistas, concibiendo la necesidad un año adicional para la formación en las especificidades de esta área de actuación. Se discuten las estrategias de formación, los campos de práctica y la estructura programática necesaria para la consolidación de esta propuesta. Se espera que este artículo pueda subsidiar la natural construcción de esta especialidad en el ámbito de la actuación médica en salud.

Palabras clave: Internado y Residencia; Servicios de Atención de Salud a Domicilio; Visita Domiciliaria; Educación en Salud; Educación Médica

\section{Introdução}

A Atenção Domiciliar (AD) tem crescido exponencialmente em todo o mundo. No subsetor público brasileiro, a AD iniciou-se com o serviço de assistência médica domiciliar e urgência (SAMDU), no Município de São Paulo, em 1949. A Internação Domiciliar (ID) no Sistema Único de Saúde (SUS) foi instituída pela Portaria 2.529/2006 e em 2011 a AD no âmbito do SUS seria definida pela Portaria 2.029, determinando 3 modalidades de cuidado prestados por equipes de Atenção Básica (eAB) e de AD. Com a portaria 2.527, de outubro de 2011, a AD ganha o status de nível de atenção à saúde focado na desospitalização, especialmente para cuidados a situações crônicas agudizadas ou agudas, reabilitação e nos cuidados paliativos. ${ }^{1-4}$

No Brasil, a AD é prestada por diversos serviços ofertados pelo SUS, como nas 39.872 equipes de Saúde da Família (eSF), presentes em 40.044 Unidades Básicas de Saúde (UBS) brasileiras, responsáveis por uma cobertura de $123.126 .735(59,74 \%)$ brasileiros ou nas 629 Equipes Multiprofissionais de Atenção Domiciliar (EMAD) e 347 Equipes Multiprofissionais de Apoio de Atenção Domiciliar (EMAP) implantadas até março de 2017 , cobrindo $54.152 .440(26,27 \%)$ brasileiros em todas as regiões do país. ${ }^{5}$ O Cadastro Nacional de Estabelecimentos de Saúde registra 515 "Serviços de Atenção Domiciliar Isolado (Home Care)" e mais 25 "Unidades de Atenção em Regime Residencial”. ${ }^{6}$

Incluem-se diversos outros serviços ligados a AD nos âmbitos municipal e estadual, como o Programa Acompanhante de Idosos (PAI), Acompanhante de Pessoa com Deficiência (APD), além de vários Serviços de Internação Domiciliar por ciclos de vida (neonatal, infantil, idoso) ou por problemas de saúde (AIDS, sequelas de hanseníase, dentre outros).

A AD destina-se a pacientes que apresentam: quadro clínico estável com restrição ao domicílio, seja permanente ou temporária, ou com algum grau de vulnerabilidade que impossibilite o atendimento ambulatorial, assim tendo como objetivo principal maior autonomia, vínculo entre paciente, família, cuidador e equipe de saúde, proporcionando tratamento, paliação, reabilitação, prevenção de agravos da saúde.

A AD no SUS se organiza em três modalidades de cuidados baseadas no nível clínico e funcional, necessidade de atenção e uso de equipamentos: ${ }^{7}$

- $\quad A D$ 1, de responsabilidade das eAB e eSF, pacientes com problemas de saúde, impossibilidade de comparecerem a serviços de maneira temporária ou definitiva e frequência de cuidados dentro da capacidade de abrangência dessas equipes; 
- $\quad A D$ 2, de responsabilidade das equipes do Programa Melhor em Casa (EMAD e EMAP). Os pacientes são elegíveis nesta modalidade nas situações:

- afecções agudas ou crônicas agudizadas, com necessidade de cuidados intensificados e sequenciais, como tratamentos parenterais ou reabilitação

- afecções crônicas-degenerativas, considerando o grau de comprometimento causado pela doença, que demande atendimento no mínimo semanal,

- necessidade de cuidados paliativos com acompanhamento clínico no mínimo semanal, com o fim de controlar a dor e o sofrimento do usuário ou;

- $\quad$ prematuridade e baixo peso em bebês com necessidade de ganho ponderal

- $\quad A D 3$, de responsabilidade das EMAD e EMAP. Os pacientes são elegíveis se apresentam qualquer das situações listadas na modalidade AD2, quando necessitarem de cuidado multiprofissional mais frequente, uso de equipamentos, como oxigenoterapia e ventilação mecânica, ou agregação de procedimentos de maior complexidade, como paracentese de repetição, nutrição parenteral e transfusão sanguínea.

Da mesma maneira, estariam inelegíveis pacientes com necessidade de monitorização contínua, assistência contínua de enfermagem, propedêutica complementar de maior complexidade ou frequência, tratamento cirúrgico em caráter de urgência ou em ventilação mecânica invasiva, quando a equipe não estiver apta para garantir a segurança do paciente em seu domicílio. ${ }^{8}$ Nestes casos específicos, o cuidado hospitalar seria uma opção mais viável, sem perder de vista a responsabilidade territorial das eAB e eSF por estas pessoas. ${ }^{1,7,9}$

Os serviços de AD têm funcionado quase que como observatórios das redes de atenção, fazendo a articulação entre a origem do paciente e seu destino, tanto da Atenção Primária à Saúde (APS) para o hospital, quanto da desospitalização para casa, o que garante uma visão ampla das demandas, e a transição do cuidado com longitudinalidade. AAD representa uma real possibilidade de mudança no modelo técnico assistencial, com uma vertente racionalizadora (como forma de diminuir custos por meio da abreviação ou substituição da internação hospitalar) e uma que pretende a reordenação tecnoassistencial com valorização da rede substitutiva de cuidados (como alternativa mais humanizada e contextualizada de acordo com as necessidades dos usuários e suas famílias). ${ }^{10}$

Sua necessidade se evidencia pela transição demográfica e epidemiológica: o aumento da expectativa de vida da população idosa e suas doenças crônicas, os avanços na atenção perinatal e intensivista neonatal, e a atenção a causas externas, como lesões traumáticas, aumentaram substancialmente o número de pessoas com demandas de cuidados relacionados à dificuldade de acesso a serviços e necessidade de cuidados intensivos contínuos. Ao mesmo tempo, é necessário desocupar leitos hospitalares, bem como leitos de espera e observação em serviços de urgência. Diante desse novo paradigma, os cuidados domiciliares se apresentam como substitutos à internação hospitalar de longa permanência, reduzindo o período de permanência no hospital e os gastos públicos. ${ }^{11}$ 
Além da desospitalização, a AD justifica-se pela vantagem de evitar exposição a patógenos multirresistentes de origem hospitalar, pelo conforto do paciente em ser cuidado em seu domicílio, e não em um ambiente inóspito e regulador de horários e hábitos diários, pela redução de custos advinda deste processo, ou pela ampliação da sobrevida de pacientes com problemas de saúde incapacitantes ou de cuidados prolongados, que passariam a viver em âmbito hospitalar ou outras instituições de longa permanência caso o domicílio não se estabeleça como local para o cuidado. Soma-se a isto a evolução tecnológica, capaz de levar ao domicílio procedimentos, exames complementares e instrumentos de monitoramento dos pacientes antes restritos ao hospital. ${ }^{1}$

Assim, há necessidade de se contar com profissional habilitado com perfil adequado ao trabalho multiprofissional e interdisciplinar da AD. Mesmo no domicílio, médicos e enfermeiros continuam a ser estratégicos e nucleares (na definição do plano terapêutico, pois são eles que prescrevem os cuidados a serem administrados, na hierarquização e classificação de complexidade do cuidado) como o são em outras modalidades de assistência à saúde..$^{10}$

Tendo em vista as demandas de formação específica em AD, este artigo discute a necessidade e o perfil de formação médica.

\section{Arcabouço Teórico}

A Comissão Mista de Especialidades (CME) é vinculada ao Conselho Federal de Medicina (CFM) e tem a atribuição de definir especialidades médicas, através de título de especialista concedido pelas sociedades de especialidades, por meio da Associação Médica Brasileira (AMB), ou pelos programas de residência médica credenciados pela Comissão Nacional de Residência Médica (CNRM). ${ }^{12}$

ACME é composta por: dois representantes da CNRM - um do Ministério da Saúde e um do Ministério da Educação - dois representantes do CFM e dois representantes da AMB. É responsável por definir por consenso as competências para sua atuação e as regras de seu funcionamento, por meio de atos específicos, e é disciplinada até o presente momento pela portaria CME Nº1/2016 anexa à Resolução CFM N².148/2016.

Assim, a definição de especialidade médica e área de atuação vigentes são:

"Especialidade médica como núcleo de organização do trabalho médico que aprofunda verticalmente a abordagem teórica e prática de segmentos da dimensão biopsicossocial do indivíduo e da coletividade.

Área de atuação - modalidade de organização do trabalho médico, desenvolvida por profissionais capacitados para exercer ações médicas específicas, sendo derivada e relacionada com uma ou mais especialidades"13

O reconhecimento de especialidades médicas tem como critérios a complexidade dos problemas de saúde e acúmulo de conhecimento em determinada área de atuação médica que transcenda o aprendizado do curso médico e de uma área raiz em um setor específico, a relevância epidemiológica, uma demanda social definida e ter complexidade que demande um conjunto de conhecimentos, habilidades e atitudes 
que exija um período mínimo de dois anos de formação, além de reunir conhecimentos que definam um núcleo de atuação própria que não possa ser englobado por especialidades já existente.

Critérios para o reconhecimento de "área de atuação" não são detalhados na resolução, mas determina-se que somente as entidades integrantes da CME são legitimadas para solicitar o reconhecimento de novas especialidades médicas e/ou áreas de atuação, e que deliberação e decisão sobre pedido de reconhecimento destas devem ser aprovadas por unanimidade pelos componentes da CME.

Em julho de 2017, a Resolução do CFM 2.162/2017 e a portaria CME no 01/2017 aprovaram a relação de 55 especialidades médicas e 59 áreas de atuação aprovadas pela CME. Essa lista vem mudando constantemente, uma vez que após alguns anos de trabalhos suspensos, há vários processos de reconhecimento de novas especialidades e áreas de atuação em discussão. A lista foi atualizada com a recente criação das especialidades Cirurgia Oncológica e Oncologia Clínica, e a Oncologia Pediátrica como área de atuação da Oncologia Clínica, da Hematologia e Hemoterapia e da Pediatria e a extinção da Cancerologia. ${ }^{14}$

Uma formação focada na AD já apresenta elementos suficientes dentro da teoria do conhecimento para se constituir enquanto especialidade médica. A prática médica no domicílio já ocorre em diversos serviços e equipes dos subsetores público e suplementar nacionais, exercida por médicos gerais (MFC, pediatras, internistas, cuidados paliativos), além da atenção domiciliar realizada por diversos especialistas a partir da clientela privada, em especial, os geriatras. Ao longo da construção dos serviços, um rol importante de competências específicas já se desenvolveu, contemplando elementos que não são aprendidos em uma única especialidade,$^{15}$ além de habilidades que hoje são desenvolvidas integralmente apenas no campo da $A D$, como uma abordagem ampla do cuidador, as especificidades de procedimentos e tratamentos realizados no âmbito domiciliar, ou o foco em tecnologias assistivas caseiras.

As especialidades médicas reconhecidas não se estabeleceram por motivos epistemológicos, mas sim por fatores políticos, sociais, econômicos e tecnológicos que não guardam relação com a teoria do conhecimento. Classificam-se pelas características dos pacientes (pediatria, clínica médica, obstetrícia, ginecologia, geriatria), partes do corpo (dermatologia, ortopedia, cardiologia), doenças ou condições (alergologia, oncologia, hansenologia), técnicas de tratamento (cirurgia, psiquiatria) e relação com máquinas especiais (radiologia, patologia clínica), às quais somam-se mais recentemente as especialidades de níveis de atenção à saúde (MFC, medicina intensiva, medicina paliativa, medicina emergencista). Nenhuma destas representa categorias epistemológicas primárias e, portanto, a medicina é derivativa, secundária e aplicada. ${ }^{16}$

$A A D$, enquanto área de atuação, deriva-se de características específicas de pacientes, ligadas a limitação de acesso, desfechos de agravos, e guardam correlação com níveis de atenção. As indicações do cuidado no domicílio estão mais ligadas ao grau de funcionalidade que propriamente a uma doença específica, o que torna ainda mais específicos os aspectos do cuidado, assim como ocorre com a MFC e a Medicina Intensiva.

Da mesma forma que a MFC enfrenta desafios quanto aos limites para a sua prática em termos de procedimentos técnicos, a AD se constrói com conhecimentos e habilidades da APS, hospital, urgência e emergências, cuidados intensivos e paliativos, mas com um diferencial. AAD se mostra menos inespecífica 
que a própria MFC, já que há menos sobreposição de saberes com subespecialidades devido a um elemento bem claro: o domicílio. Cabe à $A D$ executar procedimentos no local de vida das pessoas, entendendo que a limitação do acesso a serviços de saúde se traduz em novas formas de cuidar. ${ }^{17}$ Enquanto campo de saber, definir seus limites se correlaciona mais com as relações políticas entre as sociedades profissionais e tem mais a ver com a organização de instituições e companhias de seguros do que propriamente com o conhecimento. ${ }^{16}$

Ainda sob este aspecto, ressalta-se que, assim como a demanda crescente por saberes específicos trouxe a demanda de formação em Medicina Intensiva, a AD apresenta-se em ampliação há décadas. Especialmente no Brasil, o programa Melhor em Casa trouxe grande expansão do subsetor público nos últimos cinco anos com o surgimento das políticas de $A D$, enquanto o subsetor suplementar tem se beneficiado em especial do gerenciamento de casos no domicílio e programas específicos (como ventilação mecânica domiciliar) para a redução de custos com melhores indicadores assistenciais. ${ }^{7,11}$

A proposta de construção da residência complementar em Atenção Domiciliar como área de atuação representa a natural evolução da estruturação desses serviços de saúde e das necessidades assistenciais. Cuidados efetuados nas casas das pessoas já foram a principal forma de contato entre médicos e seus pacientes, mas ao longo do tempo, consultórios e ambulatórios, bem como estruturas hospitalares assumiram tal papel, o que impactou negativamente na formação de médicos para a AD. ${ }^{18}$ Com o recrudescimento desta modalidade de atenção, este problema tende a se agravar.

Destaca-se ainda que o domicílio representa limitações e riscos específicos como: quedas, lesões por pressão, trombose, broncoaspiração, oportunizadas pela AD que tornam sua prática empírica e aplicada. As ações realizadas neste âmbito não são parte expressiva da formação em nenhuma especialidade, e seu aprendizado ocorre no leito domiciliar, junto ao paciente e família, ao invés de ser trabalhado em pós-graduações médicas, fator agravado pela recente ampliação do mercado de trabalho médico nesses serviços.

Além disso, ao realizar procedimentos em domicílio sem uma retaguarda institucional, o médico domiciliar atua para evitar internações e deslocamentos de pessoas que já estão normalmente restritas e com isso teriam dificuldades de acessar o serviço. Mas como não há uma formação específica neste sentido, falta muitas vezes segurança jurídica para embasar esta prática, justamente pela ausência de uma titulação específica que certifique a capacidade deste especialista em realizá-lo.

Trata-se de momento profícuo ao debate sobre a criação da $A D$ como área de atuação, porém o processo deverá contar com a articulação e debate intenso com as sociedades de especialistas afins, AMB, CFM e CNRM, para obter consenso progressivo em relação ao conteúdo das ações médicas específicas das especialidades. Para isso, dentro da Medicina de Família e Comunidade (MFC) se apresentam como estratégicos:

1. Criar um programa de formação na área de atuação de $A D$, optativo em programas de MFC que reúnam condições específicas como expertise de preceptoria, campos de prática reconhecidos como de excelência, fortalecendo as experiências exitosas já em curso;

2. Valorizar estágios de graduação em MFC como primeiro contato dos graduandos em medicina com o domicílio enquanto locus privilegiado e natural para atuação do MFC; 
3. Fomentar a produção técnico-científica que forneça o aporte teórico e de pesquisa na área, aumentando o número de publicações dentro da especialidade MFC e ampliando a criação, organização e participação em eventos interdisciplinares e de outras especialidades médicas, divulgando as competências do MFC para AD;

4. Articular lideranças e entidades médicas em torno do debate; propor ao Conselho Federal de Medicina a criação de uma Comissão Temática/Grupo de Trabalho/Câmara Temática interdisciplinar sobre Atenção/Prática Médica/Cuidado Domiciliar que discuta a taxonomia, a epistemologia e as competências necessárias.

\section{Experiências de formação em atenção domiciliar}

Têm-se ampliado as iniciativas de incluir a temática da AD em currículos de graduação em saúde, e especialmente de pós-graduação. Embora ainda tímidas, as iniciativas de inserção do aluno desde o início do curso na APS faz com que o mesmo tenha contato com as Visitas Domiciliares (VD) no âmbito da AD1. A obrigatoriedade de carga horária do internato de APS ao final do curso de medicina deve ampliar esse primeiro contato que, no entanto, é incipiente e apenas demonstra que o domicílio pode ser um local de práticas terapêuticas, limitando-se ao primeiro nível desta modalidade de atenção.

Em 2014, durante a I Jornada de Medicina de Família e Comunidade e Atenção Domiciliar, promovida pelo Departamento de Medicina de Família e Comunidade da Associação Paulista de Medicina (APM), e pela Associação Paulista de Medicina de Família e Comunidade (APMFC), foi discutido o perfil de formação do profissional médico para a AD. As competências então discutidas encontram-se no Quadro $1 .^{15}$

Após este evento, a APMFC ampliou o debate com pacientes e cuidadores com dois seminários sobre cuidado médico e a escuta qualificada ao cuidador, e sua integração com a rede, nos eventos "Viradas da Saúde" em 2016 e 2017, o que gerou relevantes contribuições no sentido da formação médica para abordagem familiar e do cuidador.

Outras iniciativas de inserção de residentes de diversas especialidades médicas e multiprofissionais nas atividades do Programa Melhor em Casa corroboram a potencialidade deste campo de atenção na formação de profissionais da saúde.

O Programa de Residência em Medicina de Família e Comunidade da Secretaria Municipal de Saúde de São Paulo propõe a inserção do residente do segundo ano em um ciclo atenção domiciliar que inclui cenários de aprendizagem em equipes do melhor em casa e em serviços de cuidados paliativos por período de 3 meses. ${ }^{19}$

Em 2015 a Sociedade Brasileira de Medicina de Família e Comunidade (SBMFC) ${ }^{20}$ publicou o currículo baseado em competências como uma diretriz para a formação do MFC no Brasil, sendo inclusa no Campo "Atenção à Saúde" a área de competência "Atenção Domiciliar" (Quadro 2).

Com a necessidade crescente de ampliação das competência de médicos no campo da AD tanto para o setor privado quanto para o setor público, percebe-se que a proposta de currículo da SBMFC, embora seja ampla no sentido de formação do MFC para AD1, não é suficiente para a formação de um médico que atue em Serviços de AD (SAD), e que, por outro lado, a inclusão de todas as competências necessárias para este cuidado demandaria um tempo de prática que ocuparia e prejudicaria a aquisição de outras competências essenciais. 
Quadro 1. Competências do Profissional da AD definidas na I Jornada de MFC e AD. APM, APMFC, 2014.

\begin{tabular}{|c|c|c|}
\hline Habilidades & Atitudes & onhecimentos \\
\hline $\begin{array}{l}\text { 1. Protagonizar a tomada de decisões } \\
\text { complexas. } \\
\text { 2. Exercer a criatividade na prática de suas } \\
\text { atividades clínicas. } \\
\text { 3. Adaptar a melhor evidência científica } \\
\text { para um contexto real. } \\
\text { 4. Gerenciar conflitos familiares. } \\
\text { 5. Incrementar a relação equipe-família. } \\
\text { 6. Executar procedimentos com } \\
\text { equipamentos mínimos e sem estrutura } \\
\text { física ideal. } \\
\text { 7. Atender independente do ciclo de vida } \\
\text { das pessoas. } \\
\text { 8. Classificar a vulnerabilidade e a } \\
\text { funcionalidade familiar. } \\
\text { 9. Abordar a família sob o ponto de vista } \\
\text { social, clínico e sistêmico. } \\
\text { 10. Reconhecer os elementos presentes na } \\
\text { rede de apoio social. } \\
\text { 11. Apoiar o cuidador do ponto de vista } \\
\text { clínico e de aquisição de competências. } \\
\text { 12. Classificar a complexidade do cuidado. } \\
\text { 13. Lidar com situações de violência } \\
\text { familiar e comunitária. }\end{array}$ & $\begin{array}{l}\text { 1. Empatia e disponibilidade para discutir } \\
\text { situações fora do contexto da saúde. } \\
\text { 2. Humildade ao adentrar o terreno do } \\
\text { outro. } \\
\text { 3. Respeito pelas crenças e modelos } \\
\text { explicativos familiares do processo } \\
\text { saúde-adoecimento. } \\
\text { 4. Busca por consenso com a família na } \\
\text { definição de metas de cuidado. } \\
\text { 5. Discernimento para entender elementos } \\
\text { antropológicos que interfiram no cuidado. } \\
\text { 6. Observação ativa da família, do domicílio } \\
\text { e da vizinhança. } \\
\text { 7. Disponibilidade para ouvir e adaptar } \\
\text { condutas à realidade local. } \\
\text { 8. Neutralidade em conflitos familiares, } \\
\text { procurando estabelecer vias de diálogo. } \\
\text { 9. Autoconhecimento para evitar que } \\
\text { crenças pessoais ou sentimentos } \\
\text { negativos em relação aos demais atores } \\
\text { afetem a ação em saúde. } \\
\text { 10. Vínculo com a família para se configurar } \\
\text { como a fonte contínua de cuidados. } \\
\text { 11. Desprendimento para abordar a morte } \\
\text { e o luto de acordo com a crença de cada } \\
\text { familiar. } \\
\text { 12. Posicionamento claro diante de } \\
\text { situações de violência familiar. }\end{array}$ & $\begin{array}{l}\text { 1. A aplicação da melhor evidência clínica } \\
\text { científica disponível para cada quadro } \\
\text { clínico. } \\
\text { 2. A técnica ideal de procedimentos } \\
\text { comuns e suas possíveis adaptações. } \\
\text { 3. O tratamento de situações pouco usuais } \\
\text { na clínica ambulatorial. } \\
\text { 4. A determinação social da saúde e suas } \\
\text { vertentes. } \\
\text { 5. Representação gráfica, classificação e } \\
\text { abordagem familiar. } \\
\text { 6. Gerenciamento de casos complexos e } \\
\text { elaboração de planos de cuidados. } \\
\text { 7. Instrumentos de avaliação de autonomia } \\
\text { e mobilidade. } \\
\text { 8. Instrumentos de avaliação de sobrecarga } \\
\text { e abordagem do cuidador. } \\
\text { 9. Formas de construção de redes de apoio } \\
\text { social. } \\
\text { 10. Noções de antropologia para entender } \\
\text { a cultura local e transformar empecilhos } \\
\text { em soluções. } \\
\text { 11. Classificação da complexidade do } \\
\text { cuidado. } \\
\text { 12. Cuidados paliativos e a terminalidade } \\
\text { da vida. } \\
\text { 13. Óbito no domicílio e orientações } \\
\text { funerárias. } \\
\text { 14. Planejamento e gerenciamento de } \\
\text { fluxos de atuação no domicílio. }\end{array}$ \\
\hline
\end{tabular}

Fonte: Adaptado de Savassi (2016). ${ }^{15}$

Quadro 2. Perfil de competências para a formação do MFC em Cuidados Domiciliares. SBMFC, 2015.

\begin{tabular}{|c|c|}
\hline Pré-requisito (graduação) & Único: aceita o domicílio como espaço terapêutico. \\
\hline Competências essenciais & $\begin{array}{l}\text { 1. Faz abordagem do cuidador, comunicação efetiva e estímulo ao cuidado do cuidador; } \\
\text { 2. Realiza entrevista clínica e exame físico no domicílio considerando orgânico, mental, funcional e social; } \\
\text { 3. Avalia fatores do processo saúde doença no ambiente domiciliar; } \\
\text { 4. Plano de assistência sob a lógica do trabalho em equipe; } \\
\text { 5. Utiliza os recursos disponíveis na rede de atenção à saúde, assistência social e apoio comunitário; } \\
\text { 6. Conhece e sabe prevenir a trombose venosa profunda em acamados; } \\
\text { 7. Conhecimento de risco familiar e identificação de situações de violência familiar; } \\
\text { 8. Maneja os problemas mais frequentes no cuidado domiciliar; } \\
\text { 9. Aplica critérios de elegibilidade para os níveis de complexidade dos cuidados domiciliares - vigilância } \\
\text { em saúde, consultas e internação domiciliar; } \\
\text { 10. Demonstra conhecimentos nos procedimentos possíveis de serem realizados no domicílio; } \\
\text { 11. Realiza antropometria indireta no domicílio. }\end{array}$ \\
\hline Competências desejáveis & $\begin{array}{l}\text { 1. Maneja casos de violência domiciliar; } \\
\text { 2. Apoia situações de morte no domicílio; } \\
\text { 3. Realiza sondagem, debridamentos, anticoagulação e oxigenoterapia; } \\
\text { 4. Indica alimentação enteral. }\end{array}$ \\
\hline Competência avançada & Única: Realiza analgesia percutânea, paracentese e ventilação assistida. \\
\hline
\end{tabular}

Fonte: adaptado de SBMFC (2015). ${ }^{20}$ 
Por outro lado, uma análise da formação em especialidades gerais como pediatria e medicina interna aponta que também nessas há uma demanda de formação ampla, com cenários da prática diversificados, e que a inclusão da $A D$ em sua grade de estágios não será suficiente para a aquisição das competências necessárias para atuação nos SAD, com as mesmas limitações de tempo e campo da prática.

Em todos os cenários, é importante ter a noção de que o aprendizado representa uma espiral crescente de desenvolvimento de competências e que o programa de formação na área de atuação $A D$ deve oportunizar a ampliação de conhecimentos, habilidades e atitudes para aquele médico que deseja realizar sua prática no domicílio. Como egressos serão oriundos de diferentes formações, a necessidade de homogeneizar alguns saberes se traduz em diferentes estágios de acordo com este perfil.

As experiências de inserção de residentes e graduandos em cenários da AD parecem proporcionar uma grande sensibilização sobre o tema, mas são insuficientes para garantir uma real formação para o trabalho neste campo. Várias situações clínicas, sociais, culturais, familiares e do campo da assistência social e direitos humanos somente são contempladas quando o residente está inserido em um SAD e sua resolução demanda outras competências que não são apreendidas em hospitais, ambulatórios ou UBS. As competências Clínicas, em processos organizativos e em aspectos éticos, e o gerenciamento de casos são grandes núcleos de saberes do profissional em AD.

Pediatras e Médicos Internistas se beneficiariam de estágios em AD1, permanecendo parte de sua formação complementar nos cenários de APS, porém com foco no cuidado domiciliar, da mesma forma que MFC se beneficiariam de estágios em âmbitos de urgência, hospitalar e intensivista, em especial com foco em habilidades complementares.

O Núcleo de AD do Hospital das clínicas da USP, com mais de 20 anos de experiência na área, elenca as seguintes funções ao médico na AD: ter afinidade na área, ter uma formação generalista, usar adequadamente a propedêutica, ótima técnica para realização de procedimentos, avaliar ambiente e cuidadores, conhecer recursos tecnológicos domiciliares, ter capacidade de planejamento do tratamento com condutas otimizadas, monitoramento de doentes com doenças crônicas, dominar recursos de reabilitação, ter competência na comunicação com familiares, ter competência cultural e humildade, saber ter previsão de recursos em $A D$, reconhecer limites e possibilidades, desenvolver a compaixão e espiritualidade. ${ }^{21}$

Assim, tem-se avançado nessa discussão, e hoje se pode afirmar que há maturidade suficiente para se converter em uma proposta de área de atuação.

\section{Estruturação do programa de residência}

O programa de formação na área de atuação em AD deve ter um ano de duração para formação de médicos com as competências descritas no Quadro 1, acrescidas de outros temas tais como: critérios de elegibilidade e inelegibilidade para AD1 e transição para AD2/3; critérios de alta da AD e de internação hospitalar; segurança do paciente no domicílio; organização de plano de alta compartilhada com equipes da Atenção Básica; preparação do domicílio; qualificação de cuidadores; manejo das transições de ciclo de vida de paciente acamados crônicos; aprofundamento na abordagem terapêutica, reabilitadora e paliativa 
de crianças neuropatas; abordagem de adultos com deficiência e idosos em relação à sexualidade; abordagem de tecnologias assistivas caseiras e adaptações; terapêutica parenteral domiciliar; domínio de práticas da infectologia; economia da saúde; abordagem de situações comuns de urgência e emergência no domicílio; capacidade de liderança e coordenação de cuidado.

É importante que o programa contemple vários cenários de práticas, em especial centrado nos SAD para o manejo de situações diversas, idealmente em âmbito público e privado. Ademais, deve contemplar estágios em ambulatório de especialidade e rede hospitalar.

Nos espaços de prática e aprendizado que envolvem o domicílio, o residente deve ser exposto a várias complexidades de cuidado, critérios AD1, AD2 e AD3, bem como pacientes cuidados sob a lógica de serviços privados para ter a oportunidade de realizar assistência e internações domiciliares. Estagiará em diversos cenários, tais como UBS/ ESF, EMAD, Home Care, SAD dentre outros. Ainda, nestes espaços, deve atuar em equipe interdisciplinar e contextos comunitários distintos, podendo ter ações específicas junto aos membros da equipe, bem como aos cuidadores.

Os campos de estágio externos ao domicílio devem oferecer treinamento em conhecimentos específicos, tais como: pneumologia/oxigenoterapia, cuidados paliativos, gastroenterologia para manejo de ostomias, sondagens e paracenteses, cirurgia de cabeça e pescoço para manejo de traqueostomias, ambulatório de cardiologia e cirurgia vascular para manejo de anticoagulação, estomatoterapia para tratamento de feridas, enfermarias e UTI para prática de procedimentos de maior complexidade (paracentese, toracocentese, hipodermóclise, diálise peritoneal, ventilação mecânica invasiva e não invasiva, nutrição parenteral, transfusão sanguínea), entre outros.

A concentração em cada um destes serviços será determinada pela característica do serviço que oferece o programa e fundamentalmente pela formação de base do residente, dada a variabilidade das especialidades que poderão ingressar nesta área de atuação.

Assim, o programa deve respeitar as seguintes proporções:

- 50 a 60\% em cenários de prática domiciliares: intradomicílio a partir da UBS (AD1), SAD, Home Care, incluindo cuidados paliativos domiciliares;

- 30 a 40\% em cenários externos: UBS, Enfermarias (incluindo cuidados paliativos hospitalares), Unidades de Pronto Atendimento, Unidades de Terapia Intensiva, Centros de Reabilitação, Rede de Saúde Mental, dentre outras;

- 10 a $15 \%$ em atividades teóricas.

É desejável que os estágios do programa da área de atuação em AD contemplem cenários capazes de aprimorar competências nas áreas em que o residente demanda maior formação, como na abordagem de sondas e ostomias, oxigenoterapia e ventilação mecânica, cuidados paliativos, em especial para residentes vindos da MFC; assim como para residentes oriundos de áreas hospitalares a ampliação do foco na AD1, na abordagem familiar e do cuidador, e nos preceitos de longitudinalidade e coordenação do cuidado. Isso gera a necessidade de se estabelecer uma grade programática adaptável ao perfil de formação do residente egresso do R2 de diferentes especialidades. 
Estágios em campos como Unidades de Terapia Intensiva e Reabilitação são desejáveis, mas não obrigatórios, tendo em vista a necessidade de estruturação em redes de atenção à saúde com perfis variáveis. As habilidades que seriam desenvolvidas nestes âmbitos podem ser desenvolvidas no domicílio junto a equipes de AD.

Cuidado especial deve ser direcionado ao processo avaliativo, dado que se trata de novos programas que deverão ser constantemente avaliados e reorganizados. Assim, "avaliações 360" deverão considerar os conhecimento, habilidades e atitudes dos residentes, conforme demonstrado no Quadro 3:22-24

Quadro 3. Processo Avaliativo do Residente.

\begin{tabular}{|c|c|c|}
\hline AVALIAÇÃO DE CONHECIMENTOS & AVALIAÇÃO DE HABILIDADES & AVALIAÇÃO ATITUDES \\
\hline $\begin{array}{l}\text { - Portfólio reflexivo ao final de cada } \\
\text { estágio. } \\
\text { Avaliação teórica semestral, } \\
\text { idealmente centrada em situações } \\
\text { problema do cotidiano (casos clínicos } \\
\text { complexos, metodologia OSCE, } \\
\text { avaliação aberta ou múltipla escolha) } \\
\text { com avaliação-conceito. }\end{array}$ & $\begin{array}{l}\text { - Feedback do preceptor e } \\
\text { preceptoria-minuto diária } \\
\text { Instrumentos de avaliação da } \\
\text { prática clínica (Mini-cex, Guia de } \\
\text { Calgary-Cambridge, vídeo-feedback, } \\
\text { grupos Ballint, outros): mensais } \\
\text { - Autoscopia quando autorizada por } \\
\text { família e paciente. } \\
\text { Oficinas de habilidades com vídeos } \\
\text { de realização de procedimentos e } \\
\text { com a demonstração do preceptor nas } \\
\text { visitas domiciliares. }\end{array}$ & $\begin{array}{l}\text { Durante Mini-cex ou outros } \\
\text { instrumentos de avaliação da prática } \\
\text { clínica-mensais } \\
\text { - Preceptoria-minuto diária e feedback } \\
\text { diários } \\
\text { - Autoscopia quando autorizada por } \\
\text { família e paciente. }\end{array}$ \\
\hline
\end{tabular}

É importante que o programa de área de atuação em AD se dê junto a residências multiprofissionais, tendo em vista a enorme necessidade do trabalho em equipe interdisciplinar no domicílio.

O programa deve contar com ao menos um mês de estágio optativo, no qual o residente terá a oportunidade de aprofundamento de treinamento ou vivência em outros serviços conforme necessidade para sua formação. Por exemplo, citam-se possíveis estágios optativos: enfermaria de cuidado paliativos, hospices, hospitais dia, CAPS III com internação, serviços de transporte médico, enfermarias cirúrgicas e clínicas, Instituições de Longa Permanência de Idosos, dentre outros.

A estruturação dos programas na área de atuação em AD deve ser, portanto, versátil para abranger várias realidades dos serviços e diferentes especialidades que poderão cursá-lo, sem, no entanto, deixar de contemplar as características aqui descritas.

Ressalta-se que é ainda mais importante que o residente conte com apoio psicológico e psicopedagógico de toda equipe multidisciplinar no seu dia-a-dia para enfrentar os desafios na AD e evitar burnout, ansiedade e depressão, já que o residente está diante de cenários ainda mais desafiadores pautados pela insuficiência familiar, do poder público e tensões do mercado privado de saúde, lidando com situações angustiantes e muitas vezes insolúveis. ${ }^{25-27}$

\section{Conclusões}

A AD é um campo de atuação com particularidades que demandam formação específica. Tais elementos não fazem parte da grade curricular da graduação e não estão contemplados na formação de qualquer especialidade médica. Médicos domiciliares são formados no campo da prática, sem supervisão, sem delimitação das habilidades necessárias para a sua prática e sem uma titulação que certifique sua expertise. 
O domicílio é claramente definidor de competências aplicadas, um locus de atuação que define habilidades e atitudes específicas, e um rol de conhecimentos que definem esta formação da mesma forma que especialidades baseadas nas características de pacientes (cuidados paliativos), técnicas e tratamentos específicos pelas especificidades do domicílio (cirurgia e psiquiatria), e níveis de atenção (MFC, emergencistas, internistas e intensivistas).

O corpo de conhecimentos, habilidades e atitudes necessários demanda uma formação específica em $A D$, tanto pela pulverização dos conhecimentos dentre várias especialidades médicas quanto pela ausência de alguns destes conteúdos em qualquer especialidade. Soma-se a isto o tempo necessário para realizar tal treinamento, já que há pouco ou nenhum estágio domiciliar nas residências, o que demandaria um tempo considerável para a sua inclusão.

A MFC é uma especialidade que se beneficia do perfil generalista, sem limitação de atuação por ciclo de vida, o que torna o MFC um candidato natural a continuar sua formação geral com atuação centrada no campo da AD. A despeito disto, pela aderência da AD a outras áreas de atuação geral, especialmente aquelas ligadas a ciclos de vida, entendemos ser importante uma discussão sobre a formação que permita a entrada e, portanto, abranja outros especialistas gerais, como pediatras, médicos internistas, intensivistas, e geriatras, embora estes dois últimos já estejam contemplados na formação em medicina interna como pré-requisito. Assim, uma proposta de aprofundamento na formação em AD mais adequada seria como área de atuação compartilhada entre diferentes especialidades médicas e não como inerente a uma única especialidade médica.

Há hoje um acúmulo de conhecimentos da atuação no domicílio, devido à forma, tempo, e ritmo de manejo, e das complexidades inerentes a um cuidado sem retaguarda institucional e dependente de pessoas que não são profissionais de saúde, os cuidadores. Os problemas abordados no domicílio, por sua vez, não são relacionados a uma doença ou órgão específicos, mas são por definição problemas cuja complexidade exige um nível de cuidados baseado em critérios de exclusão que geram a necessidade de atuação de uma equipe multiprofissional, sendo o médico agente importante.

Por fim, a transição demográfica e epidemiológica exige novas formas de cuidado e parte dessa demanda será respondida com a ampliação dos SAD, demandando de seus profissionais médicos este conjunto de conhecimentos, habilidades e atitudes que exigem minimamente um ano de formação, além de reunir conhecimentos que definem um núcleo de atuação própria e de urgente necessidade de implantação.

\section{Referências}

1. Brasil. Ministério da Saúde. Secretaria de Atenção à Saúde. Departamento de Atenção Básica. Caderno de Atenção Domiciliar. Brasília: Ministério da Saúde; 2013.

2. Brasil. Ministério da Saúde. Portaria № 2.529 de 19 de outubro de 2006. Institui a Internação Domiciliar no âmbito do SUS. Brasília: Diário Oficial da União; 2006.

3. Brasil. Ministério da Saúde. Portaria № 2.029 de 24 de agosto de 2011. Define a atenção domiciliar no âmbito do Sistema Único de Saúde (SUS). Brasília: Diário Oficial da União; 2011.

4. Brasil. Ministério da Saúde. Portaria № 2.527 de 27 de outubro de 2011 . Redefine a atenção domiciliar no âmbito do Sistema Único de Saúde (SUS). Brasília: Diário Oficial da União; 2011.

5. Brasil. Ministério da Saúde. Sala de Apoio a Gestão Estratégica. Brasília: Portal SAGE; 2017 [Internet]. [citado 2017 Ago 19]. Disponível em: http://sage.saude.gov.br

6. Brasil. Ministério da Saúde. Cadastro Nacional de Estabelecimentos de Saúde. SCNES. Brasília: DATASUS [Internet]. [citado 2018 Fev 6]. Disponível em: http://cnes. datasus.gov.br/ 
7. Brasil. Ministério da Saúde. Portaria № 825 de 26 de abril de 2016. Redefine a atenção domiciliar no âmbito do Sistema Único de Saúde (SUS) e atualiza as equipes habilitadas. Brasília: Diário Oficial da União; 2016.

8. Brasil. Ministério da Saúde. Secretaria de Atenção à Saúde. Departamento de Atenção Hospitalar e de Urgência. Segurança do paciente no domicílio. Brasília: Ministério da Saúde; 2016.

9. Brasil. Agência de Vigilância Sanitária. Resolução RDC № 11, de 26 de janeiro de 2006. Dispõe sobre o Regulamento Técnico de Funcionamento de Serviços que prestam Atenção Domiciliar. Brasília: Ministério da Saúde; 2006 [Internet]. [citado 2017 Ago 26]. Disponível em: http://bvsms.saude.gov.br/bvs/saudelegis/anvisa/2006/res0011_26_01_2006.html

10. Silva KL, Sena RR, Seixas CT, Feuerwerker LCM, Merhy EE. Atenção domiciliar como mudança do modelo tecnoassistencial. Rev Saúde Pública. 2010;44(1):166-76. DOI: http://dx.doi.org/10.1590/S0034-89102010000100018

11. Dias MB, Savassi LCM, Nunes MRMTP, Zachi MLR. A Política Nacional de Atenção Domiciliar no Brasil: potencialidades, desafios, e a valorização necessária da Atenção Primária a Saúde. J Manag Prim Heal Care. 2015;6(1):1-7.

12. Brasil. Ministério da Saúde. Decreto № 8.516, de 10 de setembro de 2015. Regulamenta a formação do Cadastro Nacional de Especialistas de que tratam o $\S 4^{\circ}$ e $\S 5^{\circ}$ do art. $1^{\circ}$ da Lei oㅡ 6.932 , de 7 de julho de 1981, e o art. 35 da Lei oㅜ 12.871, de 22 de outubro de 2013. Brasília: Diário Oficial da União; 2015.

13. Brasil. Ministério da Saúde. Conselho Federal de Medicina. Resolução CFM № 2.148 de 22 de julho de 2016. Dispõe sobre a homologação da Portaria CME ㄲo 01/2016, que disciplina o funcionamento da Comissão Mista de Especialidades (CME), composta pelo Conselho Federal de Medicina (CFM), pela Associação Médica Brasileira (AMB) e pela Comissão Nacional de Residência Médica (CNRM), que normatiza o reconhecimento e o registro das especialidades médicas e respectivas áreas de atuação no âmbito dos Conselhos de Medicina. Brasília: Diário Oficial da União; 2016.

14. Brasil. Ministério da Saúde. Conselho Federal de Medicina. Resolução CFM №2.162, de 18 de maio de 2017. Homologa a Portaria CME № 1/2017 que atualiza a relação de especialidades e áreas de atuação médicas aprovadas pela Comissão Mista de Especialidades. Brasília: Diário Oficial da União; 2017.

15. Savassi LCM. Os atuais desafios da Atenção Domiciliar na Atenção Primária à Saúde: uma análise na perspectiva do Sistema Único de Saúde. Rev Bras Med Fam Comunidade. 2016;11(38):1-12. DOI: http://dx.doi.org/10.5712/rbmfc11(38)1259

16. Stephen GG. The Intellectual Basis of Familly Practice. Tucson: Winter Publishing; 1982.

17. Feuerwerker LCM, Merhy EE. A contribuição da atenção domiciliar para a configuração de redes substitutivas de saúde: desinstitucionalização e transformação de práticas. Rev Panam Salud Publica. 2008;24(3):180-8. DOI: http://dx.doi.org/10.1590/S1020-49892008000900004

18. Meyer GS, Gibbons RV. House calls to the elderly--a vanishing practice among physicians. N Engl J Med. 1997;337(25):1815-20. DOI: http://dx.doi.org/10.1056/NEJM199712183372507

19. São Paulo. Secretaria Municipal de Saude. Processo 2014-196. Parecer 396-2016 de 21-01-2016, Proposta Técnica de janeiro de 2017. [Internet]. 2017 Ago; [citado 2017 Ago 25]. Disponível em: http://ead2.saude.prefeitura.sp.gov.br/course/view.php?id=74

20. Sociedade Brasileira de Medicina Família e Comunidade. Lermen Junior N, Org. Currículo baseado em competências para Medicina de Família e Comunidade. Florianópolis: SBMFC; 2015 [Internet]. [citado 2017 Ago 13]. Disponível em: http://www.sbmfc.org.br/media/ Curriculo\%20Baseado\%20em\%20Competencias(1).pdf

21. Associação Paulista de Medicina. Departamento de Medicina de Família e Comunidade. Relatório II Simpósio de Medicina de Família e Comunidade "Prática Médica Domiciliar: Qualificação e Integração do Cuidado" [Manuscrito]. São Paulo: Associação Paulista de Medicina; 2017.

22. Aagaard E, Teherani A, Irby DM. Effectiveness of the one-minute preceptor model for diagnosing the patient and the learner: proof of concept. Acad Med. 2004;79(1):42-9. DOI: http://dx.doi.org/10.1097/00001888-200401000-00010

23. Brasil. Ministério da educação. Comissão Nacional de Residência Médica. Resolução № 5, de 8 de junho de 2004. Dispõe sobre os serviços de preceptor/tutor dos programas de Residência Médica. Brasília: Diário Oficial da União; 2004.

24. Lopes AC. Sem preceptor não há residência médica [entrevista]. São Paulo: Jornal do Cremesp; 2007. p. 3-4.

25. Catsicaris C, Eymann A, Cacchiarelli N, Usandivaras I. La persona del médico residente y El síndrome de desgaste profesional (burnout). Un modelo de prevención en la formación médica. Arch Argent Pediatr. 2007;105(3):236-40.

26. Nogueira-Martins LA. Qualidade de vida dos médicos residentes: revisão de estudos brasileiros. Cad ABEM. 2010;6:12-8.

27. Asaiag PE, Perotta B, Martins MA, Tempski P. Avaliação da qualidade de vida, sonolência diurna e burnout em Médicos Residentes. Rev Bras Educ Méd. 2010;34(3):422-9. DOI: http://dx.doi.org/10.1590/S0100-55022010000300012 